$\frac{12}{2-9-96} 85(1)$

UCRL-ID-122544

\title{
Power Spectrum Calculations Using the Fast Fourier Transform
}

\author{
R. McCallen
}

December 5, 1995

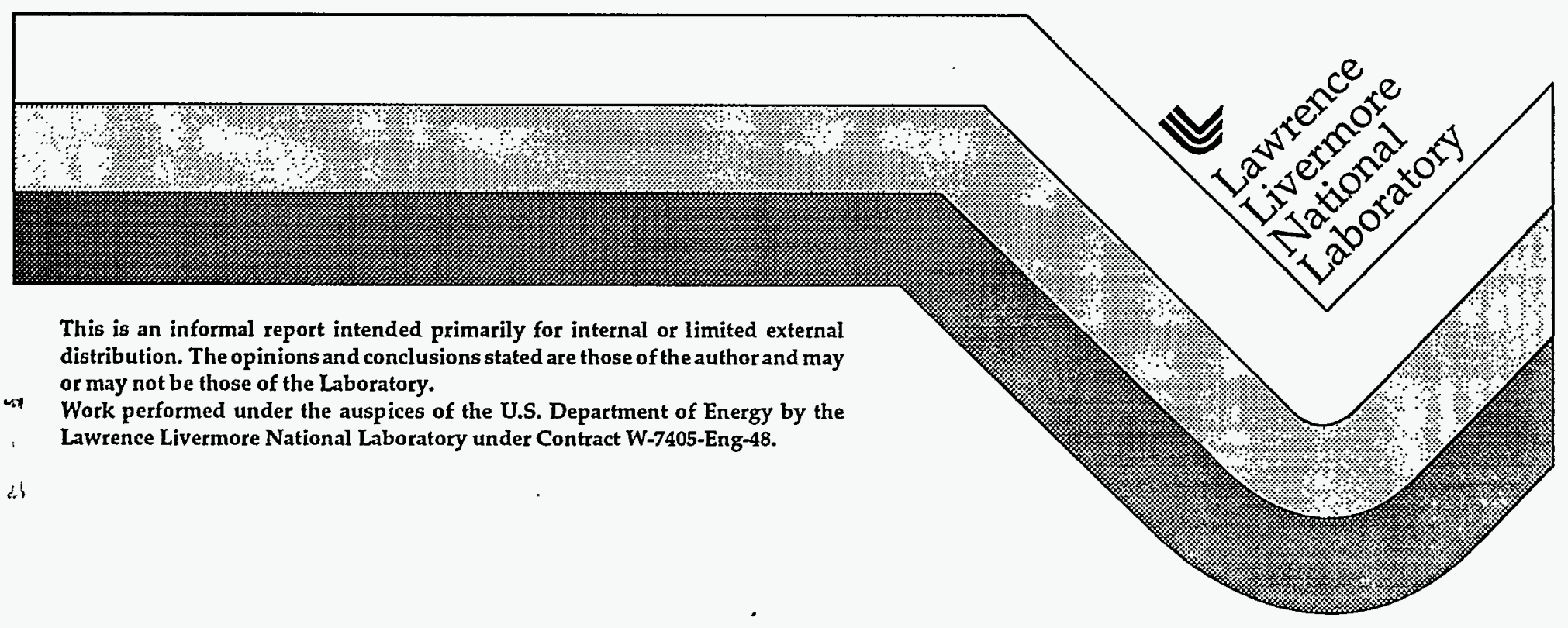




\section{DISCLAIMER}

This document was prepared as an account of work sponsored by an agency of the United States Government. Neither the United States Government nor the University of California nor any of their employees, makes any warranty, express or implied, or assumes any legal liability or responsibility for the accuracy, completeness, or usefulness of any information, apparatus, product, or process disclosed, or represents that its use would not infringe privately owned rights. Reference herein to any specific commercial product, process, or service by trade name, trademark, manufacturer, or otherwise, does not necessarily constitute or imply its endorsement, recommendation, or favoring by the United States Government or the University of California. The views and opinions of authors expressed herein do not necessarily state or reflect those of the United States Government or the University of California, and shall not be used for advertising or product endorsement purposes.

This report has been reproduced directly from the best available copy.

Available to DOE and DOE contractors from the

Office of Scientific and Technical Information

P.O. Box 62, Oak Ridge, TN 37831

Prices available from (615) 576-8401, FTS 626-8401

Available to the public from the

National Technical Information Service

U.S. Department of Commerce

5285 Port Royal Rd.,

Springfield, VA 22161 


\title{
Power Spectrum Calculations Using the Fast Fourier Transform
}

\author{
Rose C. McCallen \\ December 5, 1995
}

\section{Introduction}

In the analysis of turbulent flow, the evaluation of simulations is difficult because the results are three-dimensional and transient. We have found that analysis of the power spectra from the nodal time histories provides not only insight into the behavior of the flow, but it is a useful tool in determining the solution's spatial (grid) and temporal (time step) convergence.

We have developed a method and computer code for calculating the power spectrum for any set of equal-interval data. The code is called PWRSPEC. This report documents the method used to calculate the power spectrum, provides guidance on how to use the PWRSPEC code, and includes an example problem that was used for code validation.

\section{Method Description}

The following is a detailed description of the method used to calculate the power spectrum from tabulated data. The coded subroutines used to calculate the Fourier transform and power spectrum were obtained from Numerical Recipes by W. H. Press, B. P. Flannery, S. A. Teukolsky, and W.T. Vetterling, Cambridge Univ. Press (1989) (a book of numerical routines).

The power spectrum is an estimate of the mean squared amplitude of the time history data:

$$
\frac{1}{N} \sum_{j=0}^{N-1}\left|c_{j}\right|^{2} \equiv \text { mean square amplitude }
$$

where $c_{0}, c_{1}, \ldots, c_{\mathrm{N}-1}$ are the sampled data. The $N$-point sample at equal intervals $\Delta$ is used to compute the discrete Fourier transform

$$
C_{k}=\sum_{j=0}^{N-1} c_{j} e^{2 \pi i j k / N} k=0, \ldots, N-1
$$


using a fast Fourier transform routine. The power spectrum $P\left(f_{k}\right)$ for the zero and positive frequencies

$$
f_{k}=\frac{k}{N \Delta} \text { for } k=0,1, \ldots, \frac{N}{2}
$$

is calculated using the discrete Fourier transform as follows

$$
\begin{gathered}
P(0)=P\left(f_{0}\right)=\frac{1}{N^{2}}\left|C_{0}\right|^{2} \\
P\left(f_{k}\right)=\frac{1}{N^{2}}\left[\left|C_{k}\right|^{2}+\left|C_{N-k}\right|^{2}\right], k=1,2, \ldots,\left(\frac{N}{2}-1\right) \\
P\left(f_{N / 2}\right)=\frac{1}{N^{2}}\left|C_{n / 2}\right|^{2} .
\end{gathered}
$$

Data windowing is used to avoid data leakage. For a window function $w_{j}$, the equations for the power spectrum are

$$
\begin{gathered}
D_{k}=\sum_{j=0}^{N-1} c_{j} w_{j} e^{2 \pi i j k / N}, k=0, \ldots, N-1 \\
P(0)=P\left(f_{0}\right)=\frac{1}{W_{s s}}\left|D_{0}\right|^{2} \\
P\left(f_{k}\right)=\frac{1}{W_{s . s}}\left[\left|D_{k}\right|^{2}+\left|D_{N-k}\right|^{2}\right], k=1,2, \ldots,\left(\frac{N}{2}-1\right)
\end{gathered}
$$




$$
P\left(f_{N / 2}\right)=\frac{1}{W_{s s}}\left|D_{n / 2}\right|^{2}
$$

where

$$
W_{s s}=N \sum_{j=0}^{N} w_{j}^{2}
$$

The window function used to generate the power spectrum is called the Parzen window

$$
w_{j}=1-\left|\frac{j-\frac{1}{2}\langle N+1\rangle}{\frac{1}{2}(N+1)}\right| \text {. }
$$

To reduce the spectral variance, the data is segmented into overlapping segments. This method reduces the variance so long as the data sample has more points than needed. The total number of data points used to calculate the power spectrum is $(K+1) M$ where $K$ is the number of segments each of length $2 M$ so that the overlap of data between segments is of length $M$. To use the fast Fourier transform routine, $M$ must be equal to an integer power of 2 (i.e., $M=2^{n}$ ). Using the power spectrum calculated for each segment, an average power spectrum is generated.

This method will give the smallest variance from a fixed number of available data points. The larger the number of segments, the lower the variance. However, as mentioned above, it is assumed that there is more data than needed. For example, it was found that if the data has periodicity, it is important that each segment include several full periods. For periodic data, segmenting the data is not as important as the choice of a long segment with many cycles in each segment.

\section{Code Description and Validation}

The computer code for calculating the power spectrum using the above-described method is called PWRSPEC. It is designed to read any set of space delimited, equal interval data. The data 
must be in two columns with the independent variable (e.g., time) in the first column and the dependent variable (e.g., velocity) in the second column.

The user must input at runtime the

- file name for the file with the data to be operated on

- the total number of data points to be used $(=(K+1) M)$ and

- the number of data segments $(K)$.

The number of data segments must be an even number (i.e., $K=2 n$ for an integer $n$ ). The user must also choose $M$ to be an integer power of 2 (i.e., $M=2^{n}$ ), and obviously, $(K+1) M$ must be less than or equal to the amount of data in the specified file. The code determines the sampling interval using the first two data points in the input file. Example runtime input is given in the Appendix. The code does not yet use dynamic dimensioning, so that it is up to the user to adjust array dimensions and recompile the code.

For code validation we examine the calculated power spectrum for a series of sine functions:

$$
f(a)=\sin (a)+\sin (10 a)+\sin (20 a)
$$

shown in Figure 1. We expect the power spectrum to identify the three sine curve frequencies:

$$
\begin{gathered}
f=\frac{1}{2 \pi}=0.159 \text { for } \sin (a), \\
f=\frac{10}{2 \pi}=1.59 \text { for } \sin (10 a), \text { and } \\
f=\frac{20}{2 \pi}=3.18 \text { for } \sin (20 a) .
\end{gathered}
$$

The sampling interval for our data file is approximately 0.01047 .

The results for two different choices of data segments and number of data points are given in Table 1 and shown in Figures 2 and 3. Case I uses only $K=4$ data segments with $2 M=2 \cdot 1024=2048$ data points in each segment, using a total of $(K+1) M=5120$ data points. Every 
$2 M$ segment includes approximately $3.4(=.01047 \cdot 2048 \cdot 0.159)$ periods for $\sin (a), 34$ for $\sin (10 a)$, and 68 for $\sin (20 a)$. Thus, as expected, we are able to accurately predict the frequencies, with improved accuracy at higher frequencies.

For Case II we choose $K=10$ and $M=512$, for a total of 5632 data points. Now every $2 M$ segment includes approximately $1.7(=.01047 \cdot 1024 \cdot 0.159)$ periods for $\sin (a), 17$ for $\sin (10 a)$, and 34 for $\sin (20 a)$. As expected, we are able to predict the frequency for $\sin (10 a)$ and $\sin (20 a)$, but the data segment size of $2 M=1024$ data points is too small to accurately predict the lower frequency for $\sin (a)$.

Table 1: Predicted Frequencies for Function $\sin (a)+\sin (10 a)+\sin (20 a)$

\begin{tabular}{|l|c|c|c|}
\cline { 2 - 4 } \multicolumn{1}{c|}{} & \multicolumn{3}{|c|}{ Predicted Frequencies } \\
\cline { 2 - 4 } & $\sin (a)$ & $\sin (10 a)$ & $\sin (20 a)$ \\
\hline $\begin{array}{l}\text { Case I: } \\
K=4, M=1024\end{array}$ & 0.140 & 1.59 & 3.17 \\
\hline $\begin{array}{l}\text { Case II: } \\
K=10, M=512\end{array}$ & 0.187 & 1.59 & 3.17 \\
\hline & 0.159 & 1.59 & 3.18 \\
\hline
\end{tabular}




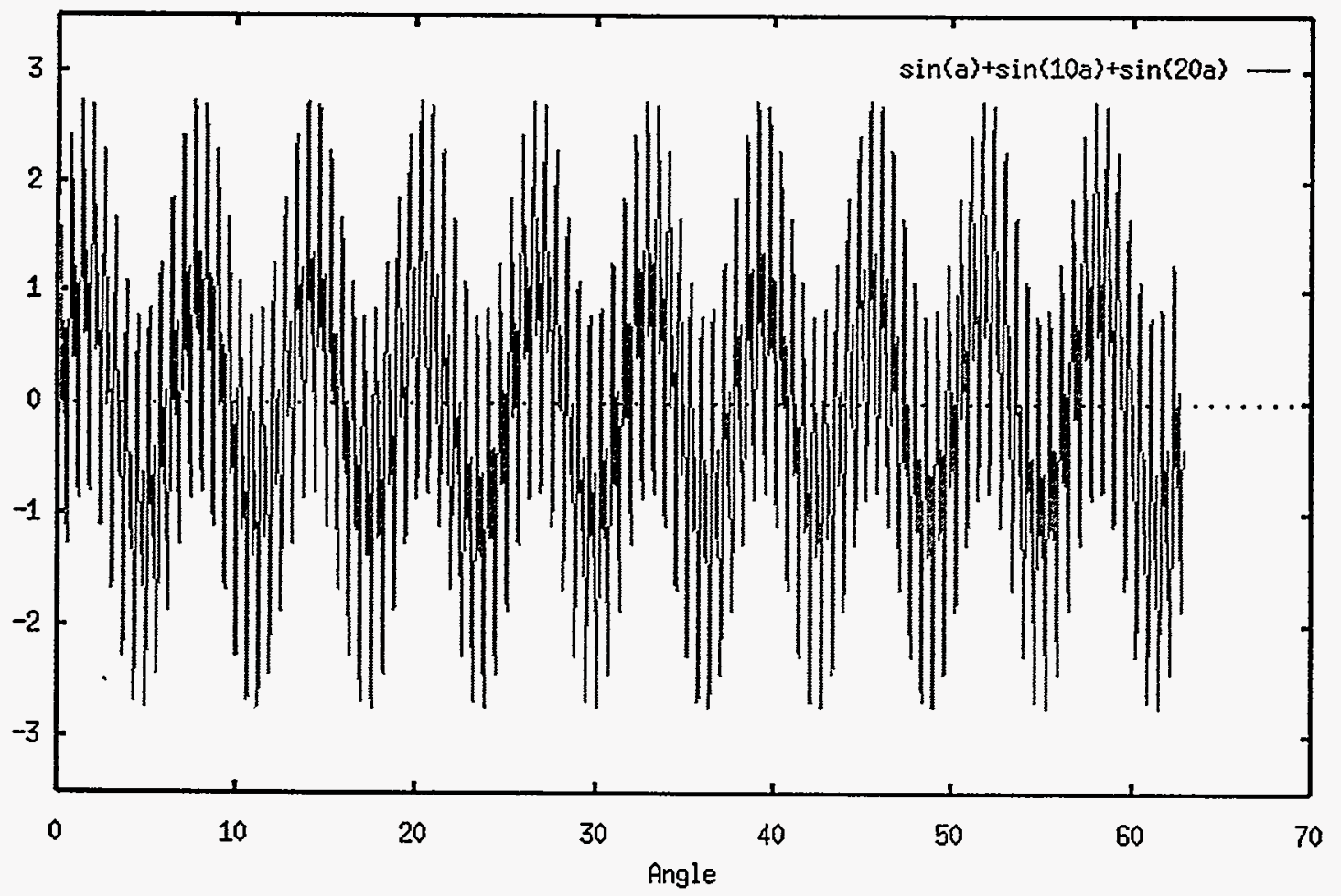

Fig. 1. Series of sine functions $\sin (a)+\sin (10 a)+\sin (20 a)$. 


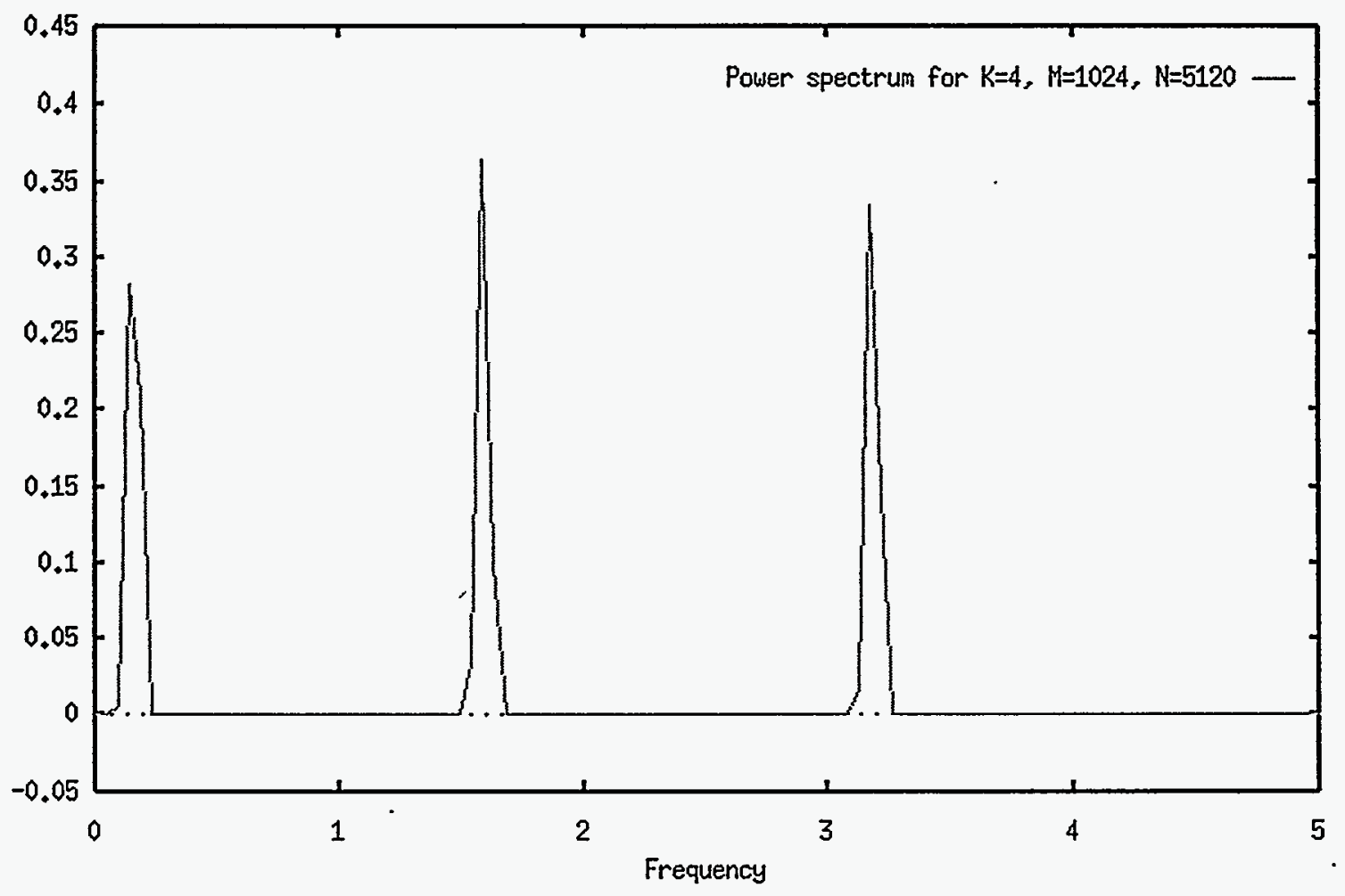

Fig. 2. Power spectrum for $\sin (a)+\sin (10 a)+\sin (20 a)$ for $K=4, M=1024, N=5120$. 


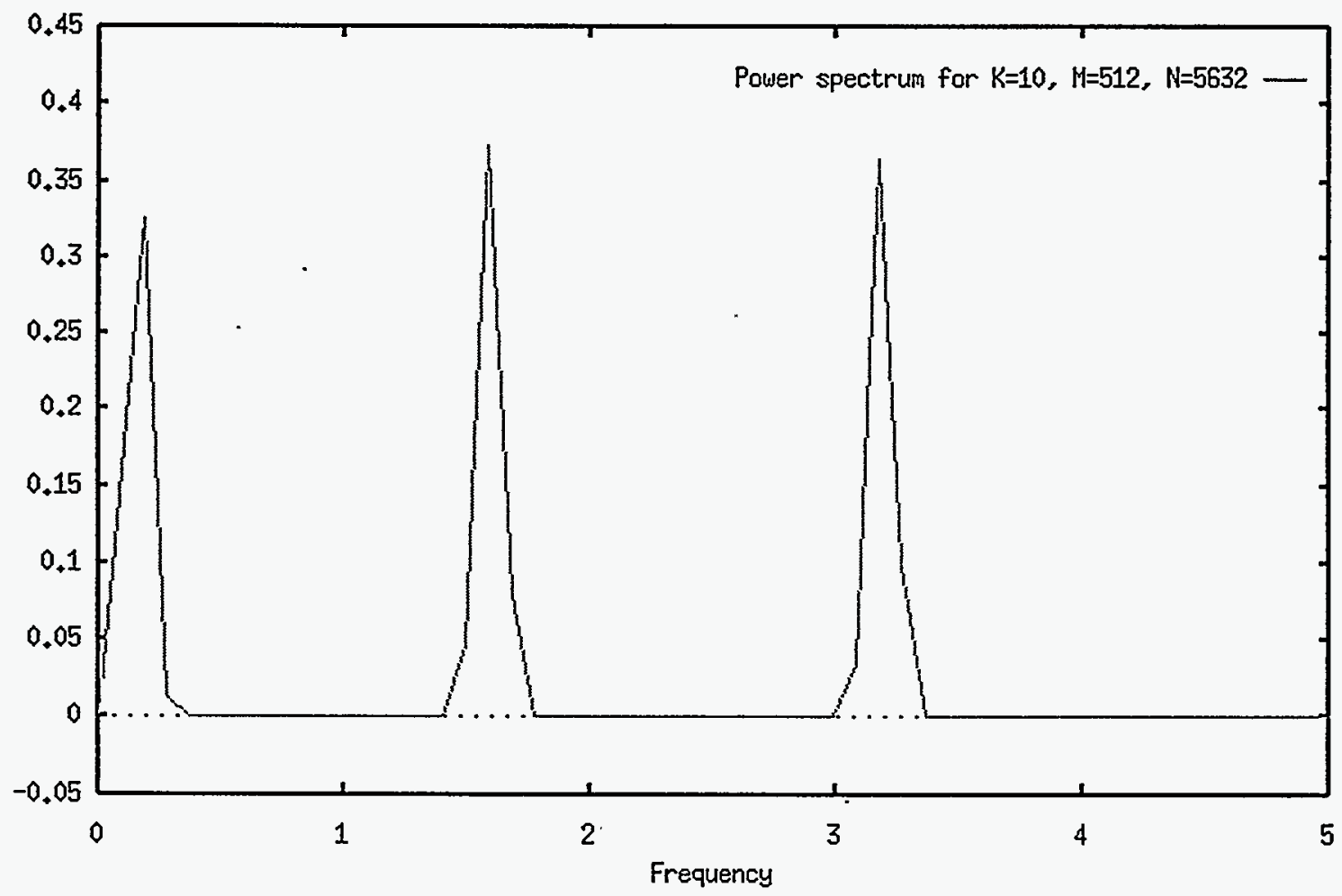

Fig. 3. Power spectrum for $\sin (a)+\sin (10 a)+\sin (20 a)$ for $K=10, M=512, N=5632$. 


\section{Appendix \\ Examples of Runtime Input}




\section{Runtime Input}

\section{Case I}

trinityo pwrspec

enter filename and number of data points to use sinecrvs 5120

input number of desired data segments

4 


\section{Runtime Input}

\section{Case II}

trinity\% pwrspec

enter filename and number of data points to use sinecrvs 5632

input number of desired data segments 10 



$$
1
$$

